\title{
Optimal timing of project control points
}

\author{
Tzvi Raz ${ }^{\mathrm{a}, *}$, Erdal Erel ${ }^{\mathrm{b}}$ \\ ${ }^{a}$ Faculty of Management, Leon Recanati Graduate School of Business Administration, Tel Aviv University, Ramat Aviv, Tel Aviv 69978, \\ Israel \\ ${ }^{\mathrm{b}}$ Faculty of Business Administration, Bilkent University, Bilkent, Ankara 06533, Turkey
}

\begin{abstract}
The project control cycle consists of measuring the status of the project, comparing to the plan, analysis of the deviations, and implementing any appropriate corrective actions. We present an analytical framework for determining the optimal timing of project control points throughout the life cycle of the project. Our approach is based on maximizing the amount of information generated by the control points, which depends on the intensity of the activities carried out since the last control point and on the time elapsed since their execution. The optimization problem is solved with a dynamic programming approach. We report the results of numerical experimentation with the model involving different types of activity intensity profiles and several levels of information loss. For each combination, we compared the optimal amount of information to the amount of information obtained with two simpler policies: control at equal time intervals, and control at equal activity contents intervals. We also investigated the effect of adding more control points on the amount of information generated. (c) 2000 Elsevier Science B.V. All rights reserved.
\end{abstract}

Keywords: Project management; Project control

\section{Introduction}

A project is an undertaking with defined objectives and established starting and ending points that needs to be completed within specified time, resources and budget constraints. Typically, prior to starting the execution of a project, a detailed plan is prepared. The plan describes the dates at

\footnotetext{
${ }^{*}$ Corresponding author. Tel.: +972-3-640-8722; fax: +972-3640-9560.

E-mail addresses: tzviraz@post.tau.ac.il (T. Raz),erel@bilkent.edu.tr (E. Erel).
}

which the activities should start and end in order to reach the objectives. It also describes the amounts of resources that should be applied towards the various activities, the budget allocated to support these resources, and the technical and operational characteristics of the deliverables to be produced by each activity.

In most cases, actual execution tends to deviate from the plan, due to a variety of factors, both internal to the project team (unforeseen difficulties, poor productivity, unexpected dependencies, etc.) and external (supplier-induced delays, price and availability variations, customer requests, etc.). Control is a mechanism designed to help cope with 
the effects of these factors. The objective of project control is to measure actual execution, compare it to the plan, analyze the deviations, and support the implementation of corrective actions to bring the project back on course.

Some of the key issues involved in designing project control procedures are how much control should be exercised, and how often. The project management literature provides some general guidance. Bent, in Cleland and King (1988, p. 579), suggests a hierarchy of control loops that includes major project milestones, quarterly performance targets and weekly performance targets. $\mathrm{He}$ further distinguishes between small projects $(<100,000$ hours) requiring control on a monthly basis, and large projects $(>1,500,000$ hours) requiring control on a weekly basis, with intermediate size projects presumably fitting in between.

Meredith and Mantel (1995) argue that control points should be linked to the actual plans of the project and to the occurrence of events as reflected in the plan, and not only to the calendar. They provide the following list of attributes that a good control system should posses: flexible, cost effective, useful, ethical, timely, accurate and precise, simple to operate, easy to maintain, and fully documented. However, they do not address the question of how to determine the extent and frequency of control needed.

Turner (1993) mentions that the frequency of the reporting period in project control depends on the length of the project, the stage of the project, the risks involved, and the organizational level of the report recipient.

Partovi and Burton (1993) carried out a simulation study to compare the effectiveness of five control timing policies: equal intervals, front loading, end loading, random and no control. Their results suggest that although there were no significant differences among the policies in the amount of cost required to recover from deviations from the plan, the end loaded policy performs best in preventing time overruns.

De Falco and Macchiaroli (1998) proposed a model for the quantitative determination of the timing of control points. Their approach is based on the definition of an effort function, which incorporates activity intensity and schedule slack aspects, and on the premise that control intensity is distributed according to a bell shaped curve around the point of maximum effort.

In this work we present an analytical framework for determining the timing of project control throughout the life cycle of the project. Our approach is based on maximizing the total amount of information generated by the control points, which depends on the intensity of the activities carried out since the last control point and on the time elapsed since their execution.

This framework is appropriate for a centralized project control mode, whereas all the participants report their progress to the project manager (or the project office). The project manager is responsible for entering the data into the planning model, carrying out actual vs. plan and forecast vs. plan analyses, and initiating corrective actions as warranted. In a decentralized control mode, where each main contributor is responsible for meeting some previously agreed deadlines for his part of the project, the framework presented here can be applied to each project part in separate.

This paper is organized as follows. We begin by introducing the model and its two key concepts: activity intensity and reporting delay. Then, we present the notation and develop the recursive equations that are used to find the optimal control timing. Next, we report the results of an experiment aimed at comparing the performance of the model to two heuristics, and study the effects of adding control points on the total amount of information generated. We conclude with a discussion of some practical implications of the model.

\section{The model}

The control cycle consists of measurement of the actual status, comparison to the plan, analysis of the deviations, and if they exists, implementation of corrective action. Measurement is a key step in the control process, since it generates the information for the subsequent. Measurement consists of determining the status of the project activities in terms of progress towards completion, costs incurred, risks, quality and performance issues, and any other relevant aspects according to the nature of the 
project. There are two aspects that determine the amount of information generated by a control point: activity intensity and reporting delay.

\subsection{Activity intensity}

Consider a project that is planned to start execution at time 0 and to complete at time $T$. We use the term activity intensity to denote the rate at which work is being done towards reaching the project objectives. If time is measured on a discrete scale, such as days, weeks, etc., then the activity intensity at time $t$ is the amount of work carried out between $t-1$ and $t$. Activity intensity can be measured in terms of person-hours, dollars, or any other measure that was used to define the project plan, and in most cases changes according to time. For an example of a mathematical form of the activity intensity function that has been validated in a variety of projects, see Murmis (1997). We will use $a(t)$ to represent the activity intensity function of the project.

We will denote by $A(t)$ the cumulative activity intensity function, representing the total amount of activity from the beginning of the project up to time $t$. Thus,

$A(t)=\sum_{x=0}^{t} a(x)$.

In order to simplify the mathematical treatment we will normalize $A(t)$ such that $A(T)=1$, meaning that at the end of the period of time planned for the project, the entire amount of effort will have been completed. Of course, at the beginning of the project no work has been accomplished, i.e., $A(0)=0$. We will also assume that $a(t) \geqslant 0$ for all $t \in[0, T]$, meaning that the cumulative amount of work accomplished can either increase with time or remain constant, but cannot decline. We will denote by $t_{m}$ the period of time at which a fraction $m$ of the work contents of the project has been completed. Thus, by definition $A\left(t_{m}\right)=m$.

\subsection{Reporting delay}

Reporting delay refers to the amount of time elapsed since the moment the activity took place up to the time of measurement. Our assumption is that data about the status of activities that took place in the past is less informative than data about recent activities. The reason for that is that as time goes by, there is a decline in the ability to analyze deviations from the project plan and to implement corrective action. We will use the parameter $p$ to denote the fraction of information lost due to the fact that measurement data about the activities that took place in a given time interval becomes available one interval later. The parameter $p$ can take values between 0 and 1 . A larger value of $\mathrm{p}$ indicates that data timeliness is more important, possibly due to higher risks, constraints, or other critical factors. If during time interval $t$ the activity intensity was $a(t)$, but the measurement is done one time interval later, then its information contents is $a(t)(1-p)$; if it is done two periods later it is $a(t)(1-p)^{2}$, and so on.

\section{Mathematical formulation}

Consider a project with a known activity intensity function $a(t)$. Based on cost, convenience and other considerations, it has been decided that there will be $n$ control points during the life of the project, which runs from 0 through $T$. The objective is to determine the timing of the control points in order to maximize the total amount of information generated. The model presented here is based upon the following assumptions:

1. Each time unit $t$ can contribute an amount of information directly proportional to the amount of activity that took place during the time unit, $a(t)$. For the sake of simplicity, we will set the proportionality constant equal to 1 .

2. For each time unit that the information is delayed, it losses a fraction $p$, as described in the previous section.

3. The amount of information generated by a control point is equal to the sum of the information contributed by all the time units since the last control point, discounted by the appropriate loss fraction. 
We now introduce the following notation:

$I(k, l)$ : Amount of information generated by a control point at time $l$, given that the last control point took place at time $k$,

$I(k, l)=\sum_{t=k+1}^{l} a(t)(1-p)^{l-t}$.

For a given $k$, increasing the value of $l$ affects $I(k, l)$ in two opposite directions: it adds more terms to the summation, but decreases the values of the existing terms, due to the fact that the exponent of $(1-p)$ increases.

$I^{*}(k)$ : Maximum amount of information that can be obtained with one additional control point, given that the last control point took place at time $k$,

$I^{*}(k)=\operatorname{Max}_{k<l \leqslant T}\{I(k, l)\}$.

$l^{*} \mid k$ : Timing of the control point that yields the maximum amount, given that the last control point took place at time $k$,

$l^{*} \mid k=\underset{k<l \leqslant T}{\arg \max }\{I(k, l)\}$.

$I_{2}^{*}(k)$ : Maximum amount of information that can be obtained with two additional control points, given that the last control point took place at time $k$,

$I_{2}^{*}(k)=\operatorname{Max}_{k<l \leqslant T}\left\{I(k, l)+I^{*}(l)\right\}$.

$I_{n}^{*}(k)$ : Maximum amount of information that can be obtained with $n$ additional control points, given that the last control point took place at time $k$,

$I_{n}^{*}(k)=\operatorname{Max}_{k<l \leqslant T}\left\{I(k, l)+I_{n-1}^{*}(l)\right\}$.

$l_{n}^{*} \mid k$ : Timing of the next control point in the sequence that yields the maximum amount, given that the last control point took place at time $k$,

$$
l_{n}^{*} \mid k=\underset{k<l \leqslant T}{\arg \max }\left\{I(k, l)+I_{n-1}^{*}(l)\right\} .
$$

The objective is to maximize $I_{n}^{*}(0)$ for a given number of control points $n$. The optimal timing of the $n$ control points is found with dynamic programming by applying the above equations recursively.

\section{Numerical experimentation}

In this section we report the results of some numerical experimentation with the model. In practice the activity intensity function $a(t)$ is derived from the specific plan for the project, and may take any shape. In order to facilitate our calculations, we chose to use the beta distribution function to represent the function $a(t)$. This choice was motivated by the fact that by selecting different values for the parameters of the beta distribution function we are able to obtain different types of activity intensity profiles: the classical bell shaped curve, front loaded and back loaded curves, the inverted bell shape, and the uniform shape. Our experiment also included several levels of steepness for each type of curve. The derivation of the parameters of the various curves is explained next.

Bell, inverted bell and flat shapes are all symmetric. Accordingly, $A(50)$ was set equal to 0.50 . Different degrees of steepness were obtained by varying the fraction of the project completed by $t=25$. Thus, $A(25)$ varies from 0.05 for the steepest bell shaped curve to 0.35 for the steepest inverted bell. Front loaded curves were obtained by completing more than $50 \%$ of the project by time 50. In this example, we set $A(50)$ equal to 0.80 , and varied the percentage completed by $t=25$, from $A(25)=0.30$ to $A(25)=0.50$. Symmetrically opposed back loaded curves were obtained by setting $A(50)$ equal to 0.20 , and varying the amount completed by $t=75$, from $A(75)=0.50$ through $A(75)=0.70$. Table 1 summarizes the characteristics of the activity intensity curves that were used in the experiment. The actual shapes of the curves appear in Fig. 1, with $t$ on the horizontal axis $a(t)$ on the vertical axis, both measured in percentage points.

The second factor that was investigated was the information loss fraction $p$. We considered three levels: $0.25,0.50$ and 0.75 . The duration of the project was set to be 100 time units, with control points possible at the end of each time unit. We assume that the information regarding the activities that take place during any given time unit becomes available at the end of the time unit. 
Table 1

Characteristics of the activity intensity curves used in the experiment

\begin{tabular}{llllll}
\hline & Shape & $A(50)$ & $A(25)$ or $A(75)$ & Mean & Variance \\
\hline A & Bell & 0.50 & $A(25)=0.05$ & 0.5 & 0.023 \\
B & Bell & 0.50 & $A(25)=0.10$ & 0.5 & 0.035 \\
C & Bell & 0.50 & $A(25)=0.15$ & 0.5 & 0.048 \\
D & Bell & 0.50 & $A(25)=0.20$ & 0.5 & 0.064 \\
E & Flat & 0.50 & $A(25)=0.25$ & 0.5 & 0.083 \\
F & Inverted bell & 0.50 & $A(25)=0.30$ & 0.5 & 0.107 \\
G & Inverted bell & 0.50 & $A(25)=0.35$ & 0.5 & 0.135 \\
H & Front loaded & 0.80 & $A(25)=0.30$ & 0.354 & 0.028 \\
I & Front loaded & 0.80 & $A(25)=0.40$ & 0.328 & 0.037 \\
J & Front loaded & 0.80 & $A(25)=0.50$ & 0.297 & 0.051 \\
K & Back loaded & 0.20 & $A(75)=0.50$ & 0.703 & 0.051 \\
L & Back loaded & 0.20 & $A(75)=0.60$ & 0.672 & 0.037 \\
M & Back loaded & 0.20 & $A(75)=0.70$ & 0.646 & 0.028 \\
\hline
\end{tabular}

For each combination of activity intensity curve and information loss fraction, we applied the dynamic programming model to find the optimal control timing, under the assumption that there will be five control points throughout the life of the project. The optimal policy was compared with two simple heuristics:

(A) Locate control points at equal intervals. With five control points, the timing will be at $t=20$, $40,60,80$ and 100. The total amount of information obtained with the five control points located according to this heuristic is equal to $I(0,20)+$ $I(20,40)+I(40,60)+I(60,80)+I(80,100)$. This heuristic altogether ignores the activity intensity function.

(B) Locate control points at equal activity content intervals. With five control points, the timing will be at $t_{0.20}, t_{0.40}, t_{0.60}, t_{0.80}$ and $t_{1}$. The total amount of information obtained with the five control points located according to this heuristic is equal to $I\left(t_{0}, t_{0.20}\right)+I\left(t_{0.20}, t_{0.40}\right)+I\left(t_{0.40}, t_{0.60}\right)+$ $I\left(t_{0.60}, t_{0.80}\right)+I\left(t_{0.80}, t_{1}\right)$. This heuristic takes into account the activity intensity function but ignores the effect of the reporting delay. It is in fact equivalent to the optimal policy with $p=0$. The timing of the five control points for each activity intensity curve according to this heuristic appear in Table 2.

The results of the experiment are summarized in Table 3. As one would expect, larger values of $p$ result in lower amounts of information for all curves and all three policies. The more interesting findings pertain to the effect that the shape of the activity intensity function has on the optimal amount of information.

Examination of Table 3 reveals that for the symmetric activity intensity functions (curves A through $\mathrm{G}$ ), the amount of information increases as the degree of steepness increases; this is true for both the bell and the inverted-bell shapes. The explanation of this observation follows from the fact that the more steep curves have regions with higher activity intensity that lead to larger amounts of information. Since $p>0$, short control intervals in these highactivity-intensity regions result in more information than in the more flat activity intensity functions. In a more flat activity intensity curve, the control points with inevitably larger control intervals generate information that is severely discounted by the positive $p$ value. In fact, the increase in the optimal amount of information is observed to be pronounced for larger values of $p$. For example, the optimal amount of information in Curve $\mathrm{A}$ is 1.85 times more than the one of Curve $\mathrm{E}$ for $p=0.25$, whereas the values are 2.20 and 2.34 for $p=0.5$ and $p=0.75$, respectively.

Among the asymmetric curves (curves $\mathrm{H}$ through $\mathrm{M}$ ), the differences in the optimal amount of information are relatively insignificant. This is apparently due to the fact that the optimal policy concentrates the control points in the 


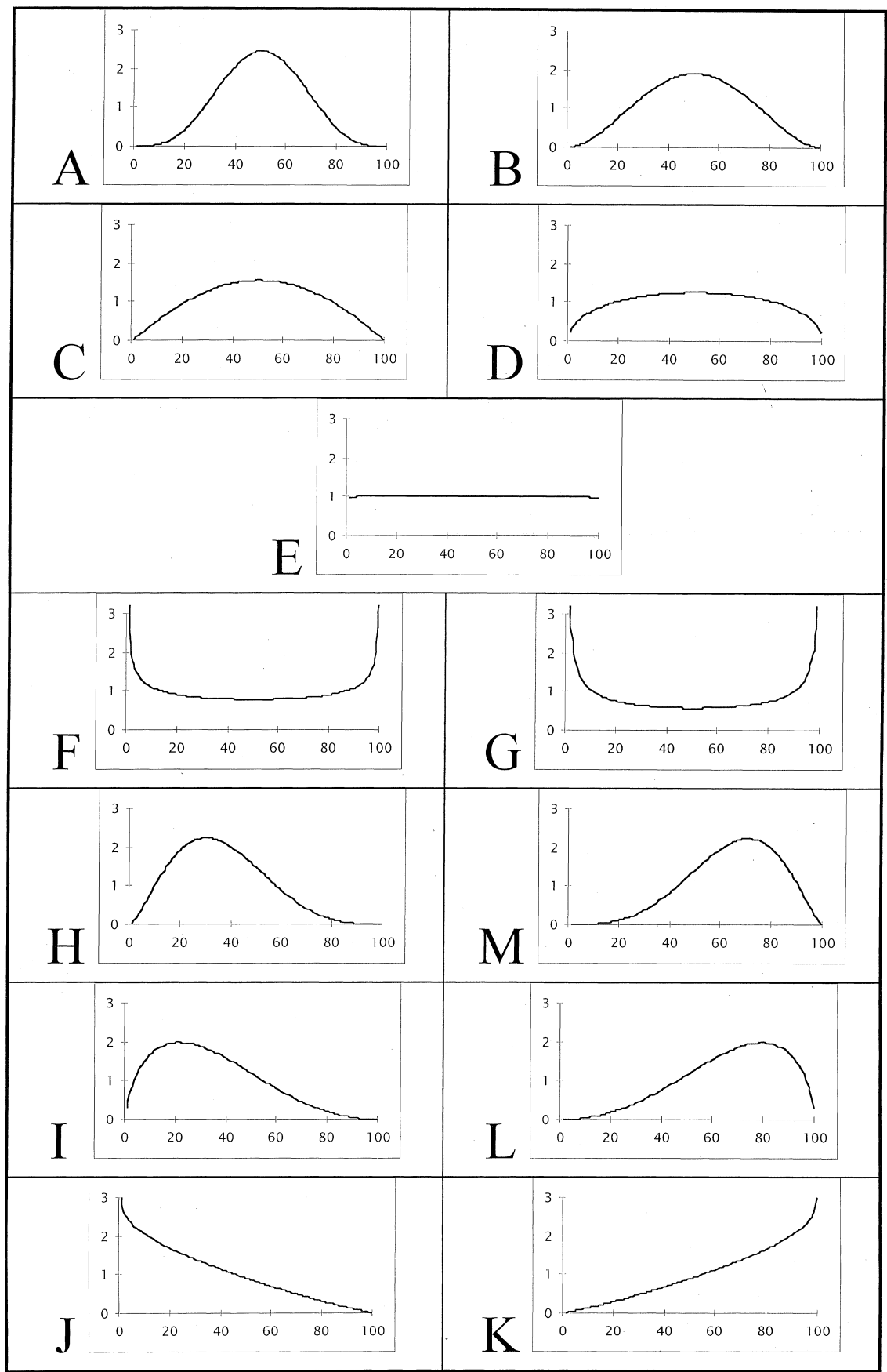

Fig. 1. Shapes of the activity intensity functions $a(t)$ used in the experiment.

high-activity-intensity regions, and there is no symmetry to force them to spread out. It is also interesting to note that the effect of front- and back-loading on the amount of information is negligibly small, especially for curves $\mathrm{H}$ and $\mathrm{M}$, and curves $\mathrm{I}$ and $\mathrm{L}$. 
Table 2

Timing of control points according to the equal work contents heuristic

\begin{tabular}{lllllllll}
\hline & Shape & $A(50)$ & $A(25)$ or $A(75)$ & $t_{0.20}$ & $t_{0.40}$ & $t_{0.60}$ & $t_{0.80}$ & $t_{1}$ \\
\hline A & Bell & 0.50 & $A(25)=0.05$ & 37 & 46 & 54 & 63 & 100 \\
B & Bell & 0.50 & $A(25)=0.10$ & 33 & 45 & 55 & 67 & 100 \\
C & Bell & 0.50 & $A(25)=0.15$ & 29 & 43 & 57 & 71 & 100 \\
D & Bell & 0.50 & $A(25)=0.20$ & 25 & 42 & 58 & 75 & 100 \\
E & Flat & 0.50 & $A(25)=0.25$ & 20 & 40 & 60 & 80 & 100 \\
F & Inverted bell & 0.50 & $A(25)=0.30$ & 14 & 37 & 63 & 86 & 100 \\
G & Inverted bell & 0.50 & $A(25)=0.35$ & 7 & 33 & 67 & 93 & 100 \\
H & Front loaded & 0.80 & $A(25)=0.30$ & 20 & 29 & 39 & 50 & 100 \\
I & Front loaded & 0.80 & $A(25)=0.40$ & 15 & 25 & 36 & 50 & 100 \\
J & Front loaded & 0.80 & $A(25)=0.50$ & 8 & 19 & 32 & 50 & 100 \\
K & Back loaded & 0.20 & $A(75)=0.50$ & 50 & 68 & 81 & 92 & 100 \\
L & Back loaded & 0.20 & $A(75)=0.60$ & 50 & 64 & 75 & 85 & 100 \\
M & Back loaded & 0.20 & $A(75)=0.70$ & 50 & 61 & 71 & 80 & 100 \\
\hline
\end{tabular}

Table 3

Total amount of information obtained with five control points under the optimal and heuristic policies

\begin{tabular}{|c|c|c|c|c|c|c|c|c|c|c|}
\hline & \multirow[t]{2}{*}{ Shape } & \multicolumn{3}{|l|}{$p=0.25$} & \multicolumn{3}{|l|}{$p=0.50$} & \multicolumn{3}{|l|}{$p=0.75$} \\
\hline & & $\begin{array}{l}\text { Optimal } \\
\text { timing }\end{array}$ & $\begin{array}{l}\text { Equal } \\
\text { time } \\
\text { intervals }\end{array}$ & $\begin{array}{l}\text { Equal } \\
\text { work } \\
\text { contents }\end{array}$ & $\begin{array}{l}\text { Optimal } \\
\text { timing }\end{array}$ & $\begin{array}{l}\text { Equal } \\
\text { time } \\
\text { intervals }\end{array}$ & $\begin{array}{l}\text { Equal } \\
\text { work } \\
\text { contents }\end{array}$ & $\begin{array}{l}\text { Optimal } \\
\text { timing }\end{array}$ & $\begin{array}{l}\text { Equal } \\
\text { time } \\
\text { intervals }\end{array}$ & $\begin{array}{l}\text { Equal } \\
\text { work } \\
\text { contents }\end{array}$ \\
\hline A & Bell & 36.94 & 19.95 & 30.91 & 22.05 & 10.01 & 16.83 & 15.63 & 6.68 & 11.29 \\
\hline B & Bell & 31.06 & 19.93 & 25.60 & 17.73 & 9.99 & 13.30 & 12.32 & 6.66 & 8.88 \\
\hline $\mathrm{C}$ & Bell & 26.78 & 19.72 & 22.17 & 14.74 & 9.79 & 11.14 & 10.11 & 6.49 & 7.39 \\
\hline $\mathrm{D}$ & Bell & 22.97 & 19.56 & 20.20 & 12.19 & 9.60 & 9.94 & 8.26 & 6.32 & 6.54 \\
\hline $\mathrm{E}$ & Flat & 19.94 & 19.92 & 19.92 & 10.03 & 9.98 & 9.98 & 6.69 & 6.65 & 6.65 \\
\hline $\mathrm{F}$ & Inverted bell & 24.63 & 21.55 & 22.37 & 16.15 & 11.89 & 12.29 & 13.37 & 8.50 & 8.76 \\
\hline G & Inverted bell & 35.44 & 25.01 & 29.66 & 28.52 & 16.19 & 18.54 & 25.88 & 12.91 & 14.35 \\
\hline $\mathrm{H}$ & Front loaded & 34.73 & 19.90 & 28.87 & 20.39 & 9.89 & 15.29 & 14.34 & 6.56 & 10.20 \\
\hline I & Front loaded & 31.81 & 18.90 & 26.11 & 18.31 & 9.21 & 13.50 & 12.74 & 6.07 & 8.97 \\
\hline $\mathrm{J}$ & Front loaded & 31.33 & 16.55 & 24.75 & 19.49 & 7.86 & 12.52 & 14.62 & 5.14 & 8.22 \\
\hline $\mathrm{K}$ & Back loaded & 33.45 & 23.91 & 31.47 & 20.56 & 12.79 & 17.23 & 15.24 & 8.79 & 11.79 \\
\hline $\mathrm{L}$ & Back loaded & 31.99 & 20.02 & 28.24 & 18.31 & 9.80 & 14.28 & 12.75 & 6.41 & 9.39 \\
\hline M & Back loaded & 34.80 & 19.61 & 29.26 & 20.39 & 9.75 & 15.27 & 14.34 & 6.49 & 10.18 \\
\hline
\end{tabular}

Another finding is that the optimal policy has a significant advantage over the two heuristics in all the activity intensity functions, expect for the case when the activity intensity curve is flat $(\mathrm{E})$. There, the two heuristics coincide with the optimal solution. The minor differences observed in row $\mathrm{E}$ of Table 3 should be attributed to the cumulative effects of rounding-off errors. Furthermore, the advantage becomes more marked as the information loss fraction $p$ increases.

From Table 3 we see that in our experiment with five control points the equal work contents heuristic is always superior to the equal time interval heuristic. To further explore this heuristic, we calculated the percent deviation from the optimal solution. The results appear in Table 4.

We notice that for the symmetric curves, the performance of the heuristic improves as the steepness of the activity intensity function decreases. This follows from the fact that the heuristic ignores the time value of information (positive $p$ ) and results in larger control intervals. For example, the control intervals in curve $\mathrm{A}$ are 9, 8,9 , and 37 , whereas in curve $\mathrm{C}$, the intervals are 
Table 4

Performance of the equal-work-contents heuristic compared to the optimum

\begin{tabular}{lllrrr}
\hline & Shape & Variance & \multicolumn{2}{c}{ Deviation from optimal solution (\%) } \\
\cline { 4 - 5 } & & & $p=0.25$ & $p=0.50$ & $p=0.75$ \\
\hline A & Bell & 0.023 & 16.32 & 23.67 & 27.77 \\
B & Bell & 0.035 & 17.58 & 24.99 & 27.92 \\
C & Bell & 0.048 & 17.21 & 24.42 & 26.90 \\
D & Bell & 0.064 & 12.06 & 18.46 & 20.82 \\
E & Flat & 0.083 & 0.10 & 0.50 & 0.60 \\
F & Inverted bell & 0.107 & 9.18 & 23.90 & 34.48 \\
G & Inverted bell & 0.135 & 16.31 & 34.99 & 44.55 \\
H & Front loaded & 0.028 & 16.87 & 25.01 & 28.87 \\
I & Front loaded & 0.037 & 17.92 & 26.27 & 29.59 \\
J & Front loaded & 0.051 & 21.00 & 35.76 & 43.78 \\
K & Back loaded & 0.051 & 5.92 & 16.20 & 22.64 \\
L & Back loaded & 0.037 & 11.72 & 22.01 & 26.35 \\
M & Back loaded & 0.028 & 15.92 & 25.11 & 29.01 \\
\hline
\end{tabular}

$14,14,14$, and 29 . The average control interval is 15.75 and 17.75 for curves $\mathrm{A}$ and $\mathrm{C}$, respectively. Note also that the control intervals in curve $\mathrm{E}$ are all equal to 20 .

In more steep curves, larger control intervals lead to a potential loss of information, whereas the optimal policy (by considering the time value of information) results in smaller control intervals with control points concentrated around the highactivity-intensity regions. Hence, as the time value of information increases (as $p$ increases), the performance of the heuristic further deteriorates.

For the asymmetric curves, the same behavior of the heuristic is observed: the performance improves as the degree of steepness of the activity intensity function decreases. Another interesting observation is that the performance of the heuristic for the back-loaded curve is always superior to the frontloaded counterpart (see rows $\mathrm{H}$ and $\mathrm{M}$, rows I and $\mathrm{L}$, and rows $\mathrm{J}$ and $\mathrm{K}$ ). This follows from the fact that the last control point of the heuristic is always at $t=100$. Having a control point at $t=100$ for the back-loaded curves does not matter, since the optimal policy also has the last control point close to the end of the project. However, the control point at $t=100$ for the front-loaded curves provides very little information, since most of the project activity takes place earlier.

As noted earlier on, the performance of the heuristic deteriorates as $p$ increases. Since a large $p$ value is associated with importance of reporting timeliness, it magnifies the difference between the heuristic and the optimal policies.

\subsection{Number of control points}

In this section we discuss the effect of the number of control points on the optimal amount of information generated. Fig. 2 depicts the increase in the amount of information resulting from adding new control points for $p=0.5$ for various activity intensity functions. In order to avoid cluttering the graph, we show the plots for five curves: one symmetric curve (A); the only flat curve $(\mathrm{E})$; one inverted bell curve $(\mathrm{G})$; one of the front loaded curves $(\mathrm{J})$; and one of the back loaded curves $(\mathrm{K})$.

The horizontal axis in Fig. 2 represents the number of control points $(n)$, while the vertical axis shows the amount of information contributed by adding one more point, that is $I_{n}^{*}(0)-I_{n-1}^{*}(0)$.

The additional amount of information as more control points are added stays almost fixed for Curve E (the flat curve), decreases almost linearly for the rest of the curves except the inverted-bell shaped curve G. The plot for curve $G$ exhibits a relatively large amount of additional information for $n=2$, and a sharp decrease followed by a almost linear decrease for $n>3$. The explanation of the above observations is as follows. For the inverted-bell case, there are two high-activity- 


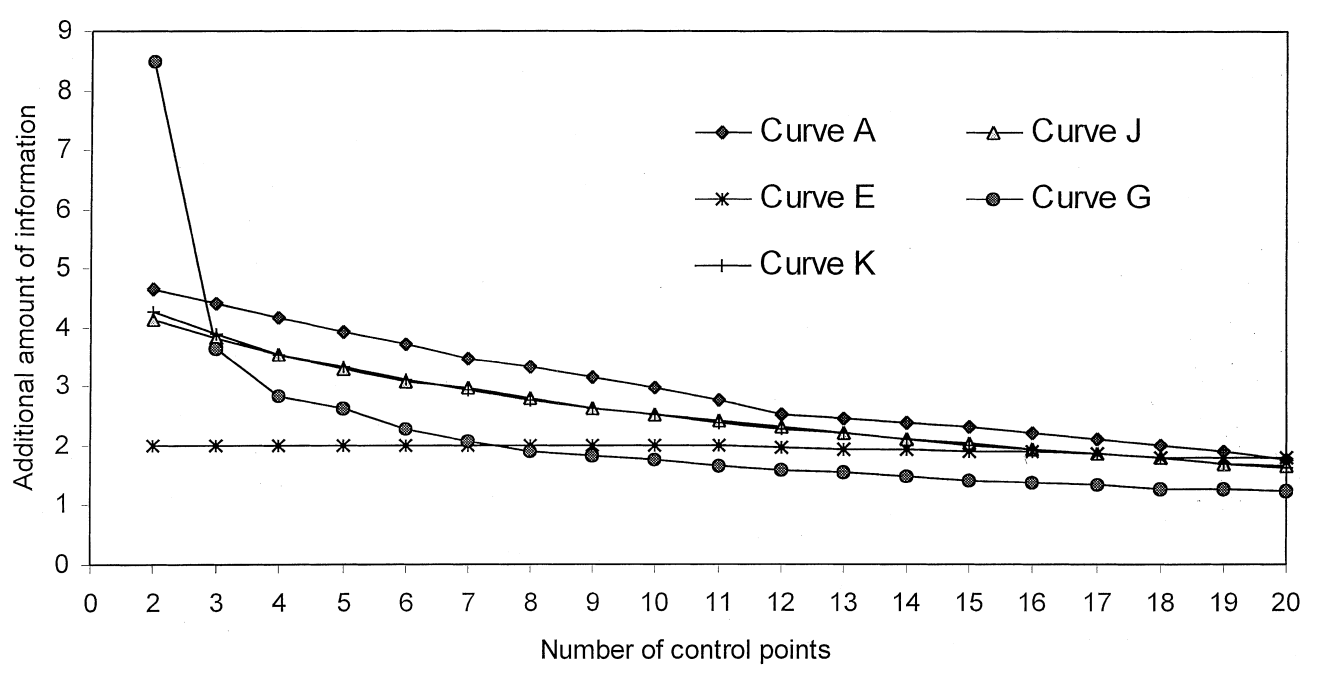

Fig. 2. Effect of additional control points on the amount of information for $p=0.5$.

intensity regions that lead to sharp decreases at the beginning. For Curve $\mathrm{G}$, the amount of information generated with one control point is 10.90 (not shown in Fig. 2); the additional amount contributed by having a second control point is 8.48 , while having a third control contributes an additional 3.64, and a fourth point contributes only 2.85 .

The sharp increase in the total amount of information generated by the second control point is attributable to the placement of the second control point to the second high-activity-intensity region. Subsequent control points are assigned to one of the two high-activity-intensity regions, which have already received some coverage and thus the potential for information contribution is much smaller.

For the other curves, the almost linear decrease is due to the saturation of the single high-activityintensity region with control points. The flat curve exhibits a very slight decrease (stays almost fixed) since it does not contain any high-activity-intensity region.

The plots for curves $\mathbf{J}$ and $\mathrm{K}$ are very close to each other. This is due to the fact that these curves are mirror-images of each other. The optimal timing of the control points for curve $\mathrm{J}$ are: 3,6 , $10,14,19$, whereas for curve $\mathrm{K}$ they are: 84,89 , 93 , 97, and 100. Thus, the first control point in curve $\mathbf{J}$ corresponds to the last control point in curve $\mathrm{K}$, the 2 nd point in curve $\mathrm{J}$ to the 4 th point in curve $\mathrm{K}$, etc. Note here that the first control point of 3 in curve $\mathbf{J}$ (instead of 0 ) corresponds to the control point of 100 in curve $\mathrm{K}$. This follows from the $I(k, l)$ calculation and this difference in the corresponding control points leads to the small difference between the plots for curves $\mathrm{J}$ and $\mathrm{K}$ in Fig. 2.

\section{Practical implications}

The basic premise of this work is that a major component of the value of control points is the information that they provide. Under this premise, a simplistic control policy that will locate control points at equal time intervals will not be effective, except for those projects that have a uniform activity intensity distribution, while policies that match control effort to activity intensity provide significantly more information. If the information loss due to reporting delay is small, the equal work contents policy will be close to optimal control schedules. However, if the time value of information is significant, then it is worthwhile to calculate the optimal policy.

Control points involve a certain cost, and consequently each additional point has to be justified in terms of the value it provides. Our analysis 
showed that the largest contribution is achieved by having one control point for each high activity time interval in the project, and that beyond that the amount of the contribution is markedly smaller and declines at a fairly constant rate. Of course, it is up to the project manager to weight the costs and benefits and to decide on the optimal number of control points.

\section{Concluding remarks}

In this paper we presented a model for optimal timing of project control while accounting for the amount of activity and the reporting delay. The limited experimentation carried out so far clearly indicates that the optimal solution is clearly superior to commonly applicable heuristics.

The solution procedure is based on dynamic programming, and is easy to implement. In fact, the calculations required for the experiment were implemented on a popular spreadsheet tool (Excel) and took a few minutes of run time for each combination. The performance of the two heuristic policies relative to the optimal policy reported depend on the shape of activity intensity curve and on the parameter $p$; the equal-work-contents heuristic is shown to perform relatively better especially for small $p$ and for flat activity intensity curves.

The structure of the problem is suitable for the dynamic programming formulation to solve large instances of the problem. On the other hand, if the objective function of the problem is modified to consider other goals and/or project-specific properties, then a more efficient heuristic may be needed. Simulated annealing approach is reported to perform well in various combinatorial optimization problems and it can be easily applied to the more complex versions of our problem. Another approach that may perform well in combinatorial optimization is genetic algorithm. The structure of our problem is again quite suitable for constructing a genetic algorithm, in particular if we want to determine simultaneously the number of control points as well as their timing.

Although in the experiment we used mathematical functions to represent the activity intensity, it should be clear that in practice the activity intensity function can take any shape. This does not limit in any way the applicability of the approach presented here. In fact, it is possible to link the output of the software used to plan the project schedule and activity profile to a module that carries out the model calculations and feeds back into the schedule the recommended dates for project control points.

The model represents a new approach to an area that has received relatively little research attention. There are several points that deserve additional work. They include investigating the effect of deviations from planned execution on the total amount of information generated by the control points; and expanding the model to include other relevant factors, such the control costs and the value of being able to modify the remaining portion of the plan.

\section{References}

Cleland, D.I., King, W.R., 1988. Project Management Handbook, 2nd ed. Van Nostrand-Reinhold, New York.

Falco, M., Macchiaroli, R., 1998. Timing of control activities in project planning. International Journal of Project Management 16 (1), 51-58.

Meredith, J.R., Mantel, S.J., 1995. Project Management - A Managerial Approach, 3rd ed. Wiley, New York.

Murmis, G.M., 1997. S curves for monitoring project progress. Project Management Journal 28 (3), 29-36.

Partovi, F.Y., Burton, J., 1993. Timing of monitoring and control of CPM projects. IEEE Transactions on Engineering Management 40 (1), 68-75.

Turner, J.R., 1993. The Handbook of Project Based Management, McGraw-Hill, New York. 